\title{
Infrastructure and Spectrum Sharing Trade-offs in Mobile Networks
}

\author{
Jacek Kibiłda \\ Trinity College Dublin \\ Ireland \\ Email: kibildj@tcd.ie
}

\author{
Paolo Di Francesco \\ Trinity College Dublin \\ Ireland \\ Email: pdifranc@tcd.ie
}

\author{
Francesco Malandrino \\ The Hebrew University of Jerusalem \\ Israel \\ Email: francesco@cs.huji.ac.il
}

\author{
Luiz A. DaSilva \\ Trinity College Dublin \\ Ireland \\ Email: dasilval@tcd.ie
}

\begin{abstract}
We assess the fundamental trade-offs between spectrum and radio access infrastructure sharing. Specifically, we analyze three mobile network sharing scenarios: radio access infrastructure sharing, spectrum sharing, and the combination of the two, which we term full sharing. We perform our analysis employing stochastic geometry models, such as the homogeneous Poisson point process and the Gauss-Poisson process, which allow us to vary the spatial correlation in base station placements of different mobile operators. Our major contributions can be summarized as follows. We derive analytical expressions for the coverage probability and the average user data rate for a shared network created as a union of independently distributed networks for each of the three sharing scenarios. For the general base station placement model, we show that infrastructure and spectrum sharing cannot be simply substituted for each other, as there exists a trade-off in the coverage and data rate performance between the two. Moreover, the combination of the two approaches does not simply produce linearly scaling gains, as the increase in the data rate is traded for a minor reduction in the coverage (when compared to infrastructure sharing performed in isolation). We show that the spatial distributions of the networks of the sharing operators have a significant impact on the performance of sharing. We show that respective density of the networks of the two operators influences how the two operators perceive sharing gains. In particular, it is often the case that a larger operator has more to gain from sharing due to disproportionally lower interference suffered from the smaller operator.
\end{abstract}

\section{INTRODUCTION}

The past two decades have witnessed world-wide expansion of mobile wireless connectivity. In many countries the mobile market penetration has surpassed $100 \%$, with the average for the EU countries at roughly $124 \%$. This success in many ways was fostered by the conventional mobile market model, which was based on exclusive ownership of both the network infrastructure and frequency spectrum licenses by the mobile network operators.

However, this model seems to have a limited remaining lifespan, as subscribers push for uncapped mobile data at lower prices, with heavily-subsidized or even free mobile devices. Yet, the cost of wireless capacity is not decreasing. In fact, the world-wide mobile CAPEX is predicted to grow from around $\$ 216$ billion in 2014 to $\$ 224$ billion by 2017, as reported during the Mobile Wireless Congress (MWC'2015). The annual fees for radio spectrum usage are also subject to a major price revision; for example, in the UK the Office for Communications (OfCom) has recently put out a proposal that will result in an increase in annual spectrum fees from $£ 64.4$ million to $£ 246.7$ million for all domestic mobile operators

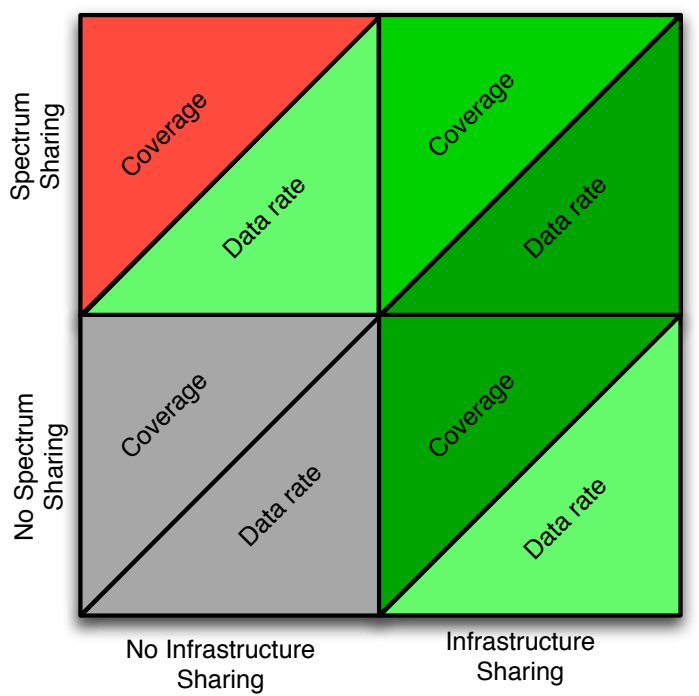

Fig. 1. Conceptual depiction of the effect of radio access infrastructure and spectrum sharing on network coverage and data rate. Each square corresponds to the network performance of a different combination of spectrum and infrastructure sharing. Each square is split evenly between network coverage and user data rate. Saturation of the green-coloured cells reflects the gain with respect to the no sharing case (grey cells), while red-coloured cells represent negative gain. Infrastructure sharing in isolation greatly improves network coverage, but provides minor gain in terms of data rate; spectrum sharing (when worst case interference scenario is considered) provides minor gains in data rate, but degrades coverage. When applied in combination, both coverage and data rate are improved over the no sharing case, with network coverage being slightly worse than in the pure infrastructure sharing case, as a result of increased interference.

combined. Moreover, the costs associated with base station sites, for example, rental, maintenance, and power, are the largest OPEX factors facing operators and are remaining static or even increasing in certain cases.

One approach for mobile operators to ease the burden of these large costs is to release ownership and control over some components of the network, for example, the radio access network or the backhaul, in the areas that generate the most costs. This may mean getting involved in network sharing agreements with other operators, and/or subletting elements of the radio access infrastructure from other infrastructure providers. Another possibility is to re-use spectrum between operators wherever and whenever capacity expansion is needed. Indeed, we are starting to gather evidence, see [1], that the spatial correlation in demand experienced by mobile operators is small enough to encourage this type of sharing. 
Both infrastructure and spectrum sharing can also be applied in combination, which should result in even larger costs reduction to the mobile operators.

The decisions on whether to share (and how) are not purely technical and involve consideration of various costs, legal/regulatory framework, operator's position in the market, corporate strategy, etc., [2]. In this paper, we are concerned with analyzing the technical aspect of these decisions only, namely the network-level performance of various mobile network sharing scenarios and the trade-offs involved with respect to the spatial distribution of the shared base stations. Fig. 1 summarizes our main results and trade-offs observed.

\section{A. Background and motivation}

Wireless network sharing can be classified as either passive or active [3]. Passive sharing happens between mobile operators that decide to share base station sites and their basic installations, such as mast, cooling equipment and power supply. Active sharing, on the other hand, involves some level of abstraction (virtualization) of the shared physical resources. These resources include physical infrastructure elements such as base stations, in which case we talk about infrastructure sharing, or time-frequency spectrum blocks, which are tied to the notion of spectrum sharing.

The key idea behind radio access infrastructure sharing is to allow the subscribers of one operator to utilize the radio access network of another operator to improve the coverage or capacity offered to those subscribers. From the network perspective, when a physical element of the radio access network is shared the available physical resources are split statically or dynamically between the sharing operators. The infrastructure sharing approach may be especially vital to improving or enabling appropriate coverage in under-served areas [4]. Commercial examples of active infrastructure sharing include Newco, the joint venture of two Danish MNOs [5] and NetWorks!, a company setup by the Polish units of T-Mobile and Orange [6].

Conventionally in mobile networks, spectrum is divided into frequency bands, with each band being exclusively reserved for use by a particular service, say GSM or LTE, and obtained by a particular operator through a spectrum auction. This allocation of bands is coordinated on the international level by the International Telecommunications Union (ITU) and is further locally implemented by regional communications regulators. From the perspective of an operator, exclusive access to spectrum allows it to have sole control over the offered quality of service, and secure a return on large-scale infrastructure investments [7]. However, from a broader perspective, exclusively licensed spectrum remains under-utilized [8], which, given low spatial correlation in mobile demand across mobile operators [1], may be a good reason to seek more elastic models of spectrum usage and licensing, such as, for example, licensed shared access [9].

Both industry and research communities recognize the importance of network sharing in the evolution of mobile networks. We have the 3GPP-defined standards for network sharing: the Multi-Operator Core Network (MOCN), where only the radio access network is shared, and the Gateway Core Network (GWCN), whereby elements of the core network are also shared [10]. We have the 2012's report from the European Commission entitled "Promoting the shared use of radio spectrum resources in the internal market". We have communications regulators testing the feasibility of various spectrum sharing models, as is the case with, for example, the recent ruling of the FCC to open military frequencies in $3550-3700 \mathrm{MHz}$ band to mobile broadband services ${ }^{1}$.

The research community has also contributed to this trend by proposing and evaluating various network sharing models. In [11], gains in spectral efficiency are evaluated for two spectrum sharing regimes: orthogonal spectrum sharing, where operators exclusively assign spectral resources coming from a shared pool, and non-orthogonal spectrum sharing, where the operators aggressively re-use spectrum by allowing more than a single operator to use a specific spectral resource in any given area at a particular time. Infrastructure sharing, on the other hand, by allowing users to freely switch between the networks of multiple operators, is shown to reduce the number of base stations required to provide mobile service and improve coverage in under-served areas [4]. Spectrum and infrastructure sharing may also be applied jointly, and in this case full benefits of network sharing are achieved when virtualized access networks are set up from a pool of virtualized physical resources such as base stations, spectrum or cloud processing units [7]. In [12] a comparative analysis of three sharing regimes (capacity, spectrum and virtualized resource sharing) is carried on a two-operator grid-deployment model. The analysis shows that capacity sharing outperforms other forms of sharing while being the most feasible shortterm solution, with no need for additional infrastructural investments, contrary to virtualized resource sharing, which requires software-defined network elements. In our work we further generalize the above studies, by focusing on the basic trade-offs between spectrum and infrastructure sharing, which we evaluate for various spatial distributions of the radio access infrastructure.

\section{B. Our approach and contributions}

In this paper, we assess fundamental trade-offs between spectrum and infrastructure sharing, and whether (or to what extent) one can be substituted for the other. Moreover, we consider the two in combination and quantify the resulting network performance gains. To achieve our goal we define three network sharing scenarios: infrastructure sharing, spectrum sharing, and full sharing (when both spectrum and infrastructure are shared). For each of the scenarios we analyze the resulting network performance in terms of coverage and user data rate. We generalize these results by considering various spatial distributions of base stations. Specifically, we look at two mobile operators whose networks have been deployed either: independently, or with clustering, i.e., there is strong spatial correlation between the locations of base stations of one operator and the locations of base stations of the other operator. We also consider an asymptotic case of clustering, i.e., colocation, where the nearest base stations of two operators are located an arbitrarily small distance apart. The clustering case is of particular interest as it is representative of real radio access network deployments, which tend to be deployed to

\footnotetext{
${ }^{1}$ The ruling can be accessed here: http://transition.fcc.gov/Daily_Releases/ Daily_Business/2015/db0421/FCC-15-47A1.pdf
} 
follow social and geographical features, resulting in clustered deployment patterns, see [13], [14].

We perform our analysis by applying stochastic geometry [15]. The key motivation is to analyze the network performance over various realizations of a network consisting of base stations which follow a particular spatial structure. This structure may be expressed as a stochastic model so that the spatial distribution of nodes resembles that of a real radio access network, see, for example, [14], [16]. In particular, we apply the superposition of two homogeneous Poisson point processes (hPPP) [15], to model independently distributed networks, and the Gauss-Poisson process (GPP) [15], to model clustered networks. In the former case, we derive analytical expressions describing the coverage probability and the average user data rate in interference-limited mobile networks for the three sharing scenarios outlined above. Whenever finding useful closed-form expression is not possible, as is the case with the GPP model, we rely on Monte Carlo methods to numerically obtain the desired performance metric.

Our major contributions can be summarized as follows:

- We derive analytical expressions for the coverage probability and the average user data rate for a shared network created as a union of $|\mathcal{N}|$ networks distributed according to the homogeneous Poisson point process for the three scenarios of infrastructure, spectrum, and full network sharing.

- We show that infrastructure and spectrum sharing cannot be simply substituted for each other, as there exists a trade-off in coverage and data rate between the two. Moreover, the combination of the two approaches does not simply produce linearly scaling gains, as the increase in the data rate is traded for a minor reduction in the coverage (when compared to infrastructure sharing performed in isolation).

- We show that the spatial distributions of the networks of the sharing operators have a significant impact on the performance of sharing.

- We show that the respective density of the networks of two operators influences how the two operators perceive sharing gains. In particular, it is often the case that the larger operator has more to gain from sharing due to disproportionally lower interference suffered from the smaller operator.

This paper is structured as follows: Sec. II outlines our model of a shared mobile network; Sec. III contains the description of our sharing scenarios; Sec. IV presents analytical expressions of network performance under each of the considered scenarios; Sec. V provides a set of numerical results and the discussion of those results; Sec. VI concludes the paper and provides specific suggestions for follow-up research.

\section{SYSTEM MODEL}

Wireless network as a point process We model base station deployment as a point process defined as a random countable $\operatorname{set}^{2} \Phi=\left\{x_{1}, x_{2}, \ldots\right\} \subset \mathbb{R}^{2}$, with elements being random variables $x_{i} \in \mathbb{R}^{2}$. We define $\mathcal{N}$ as the set of mobile operators, with its cardinality (the number of sharing operators) denoted as $|\mathcal{N}|$. Consequently, we denote the deployment of a single operator $i \in \mathcal{N}$ as $\Phi_{i}$, and the superposition of all individual deployments as $\Phi=\bigcup_{i \in \mathcal{N}} \Phi_{i}$. We also denote the bandwidth of operator $i$ as $w_{i}$, and, when spectrum is shared between $|\mathcal{N}|$ operators, we denote the aggregate bandwidth as $w=\sum_{i \in \mathcal{N}} w_{i}$ (the individual spectrum bands being aggregated are non-overlapping). Let us also remark that whenever calculating the data rate we represent it in nats/s. For the sake of clarity of our argument, from now on we will assume $^{3}|\mathcal{N}|=2$, which will determine the choice of a point process for modelling clustered behaviour. The obtained results are still generalizable provided that the clustered point process model is correctly refined.

Clustered deployment Base stations belonging to different mobile operators often cluster as a result of, for example, spatial variations in population density [13]. Given two operators, we model their combined infrastructure as distributed according to $\Phi$ which is a marked GPP process ${ }^{4}$. In this process, clusters of points are distributed according to the hPPP with intensity $\lambda_{p}$. These clusters consist of one or two points with probability $1-p$ and $p$, respectively. If a cluster has two points, one of the points is located in the center of the cluster, i.e., at the location of the parent point, and the other point is uniformly distributed on a circle with radius $u$ centered at the parent point.

In order to identify the base stations of different operators each point (base station) $x \in \Phi$ is also assigned a mark $m(x) \in \mathcal{N}$ which represents the operator that it belongs to. We assign the marks according to the following strategy: each point located in the center of each cluster receives operator assignment $m(x)=1$, while all the other points are assigned $m(x)=2$.

Signal-to-interference and noise ratio One of the most relevant metrics quantifying the performance of a wireless system with many concurrent transmissions is the signal-tointerference and noise ratio (SINR). It accounts for variations in the received signal power observed due to the random spatial distribution of the receivers, the random spatial distribution of the transmitters (and interferers), and stochastic processes describing channel effects such as shadowing or fading. We assume that the power of the signal received by a typical user located in the origin $(0,0)$ is affected by two factors: pathloss $l(x)$ and power fading $h_{x}$, where $x$ denotes the location of the serving transmitter. The pathloss function $l: \mathbb{R}^{2} \rightarrow \mathbb{R}_{+}$is of the form $l(x)=\|x\|^{-\alpha}$, where $\alpha$ is the pathloss exponent, and the power fading between the user and the serving transmitter $x$ is spatially independent and exponentially distributed with unit mean $\left(h_{x} \sim \exp (1)\right.$ ), i.e., we assume Rayleigh fading. That also implies unitary transmit power for all transmitters. In addition, we make the following assumptions: (i) our results

\footnotetext{
${ }^{2}$ Using the random set formalism we implicitly assume that inter-operator base stations do not occupy the same location. Yet, it does not preclude base stations to be located arbitrarily close to each other, which we consider as co-location.

${ }^{3}$ Even though our analytical results will be derived for the general case of $|\mathcal{N}| \geq 2$.

${ }^{4}$ For the case of $|\mathcal{N}|>2$, we would need to adopt, for example, one of the point processes from the family of the Poisson clustered processes [15].
} 


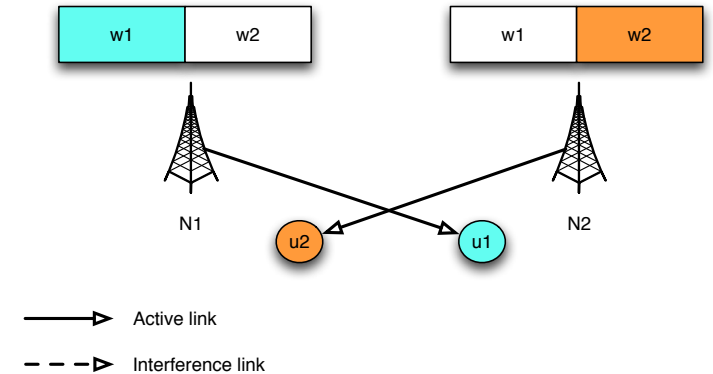

Fig. 2. Two operators (1 and 2) with subscribers $(\mathrm{u} 1, \mathrm{u} 2)$ exclusively using their infrastructure $(\mathrm{N} 1, \mathrm{~N} 2)$ and spectrum (w1,w2).

apply to the downlink only, (ii) worst-case interference is considered, i.e., all transmitters transmit simultaneously. In addition, when presenting the final results, we make the following two assumptions, which help us derive easy to interpret closedform representations: (iii) the networks are interference-limited (the noise power is ignored), which reduces the SINR to the SIR; (iv) the pathloss exponent is $\alpha=4$. These assumptions are in line with those made in similar investigations of network coverage and data rate [16], [17].

\section{REFERENCE SHARING SCENARIOS}

The conventional scenario of a commercial mobile market consists of competing operators, each of which owns the network infrastructure and holds a license to spectrum, which also implies that subscribers of one operator exclusively ${ }^{5}$ utilize the infrastructure and spectrum of their operator as depicted in Fig. 2. This serves as our baseline for comparison.

In the following we describe the sharing scenarios: infrastructure sharing, spectrum sharing and full sharing.

Infrastructure sharing scenario In the infrastructure sharing scenario we assume that operators pool their radio access infrastructure, i.e., their joint network can be represented as $\Phi=\bigcup_{i \in \mathcal{N}} \Phi_{i}$, without pooling their operational frequencies, see Fig. 3. Effectively, interference to the desired signal is identical to the single-operator case, i.e., it comes from $\Phi_{i}$ where $i$ is the operator to which the serving base station belongs. In this scenario users connect to the closest base station belonging to the network of any of the $|\mathcal{N}|$ operators. Henceforth, the SINR for this scenario takes the following form:

$$
\mathrm{SINR}=\frac{h_{x} l(x)}{W+\sum_{y \in \Phi_{i} \backslash\{x\}} h_{y} l(y)},
$$

where $W$ is the noise power.

Spectrum sharing scenario In the spectrum sharing scenario we assume that operators pool their spectrum, i.e., their aggregate bandwidth is $w=\sum_{i \in \mathcal{N}} w_{i}$, without sharing their infrastructure, see Fig. 4. Effectively, interference to the desired signal comes from base stations of all operators, i.e., $\Phi \backslash\{x\}$, while the subscribers of operator $i$ may connect only to the closest base station in $\Phi_{i}$. From the perspective of interference this scenario may be considered as the worst-case non-collaborative spectrum sharing scenario, i.e., operators do

\footnotetext{
${ }^{5}$ Leaving international roaming out of the picture.
}
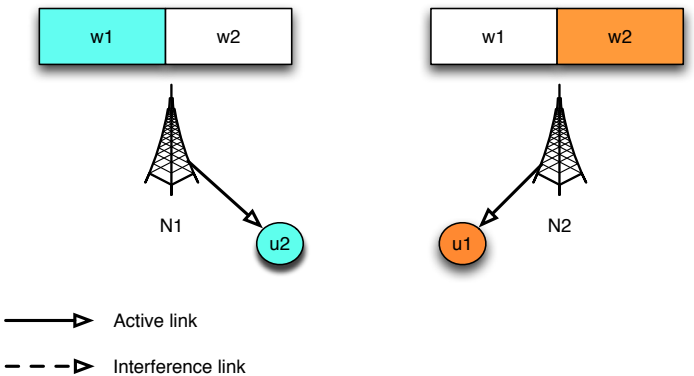

$--\longrightarrow$ Interference link

Fig. 3. Two operators (1 and 2) with subscribers (u1,u2) exclusively using their spectrum $(\mathrm{w} 1, \mathrm{w} 2)$, yet, using shared infrastructure $(\mathrm{N} 1, \mathrm{~N} 2)$.

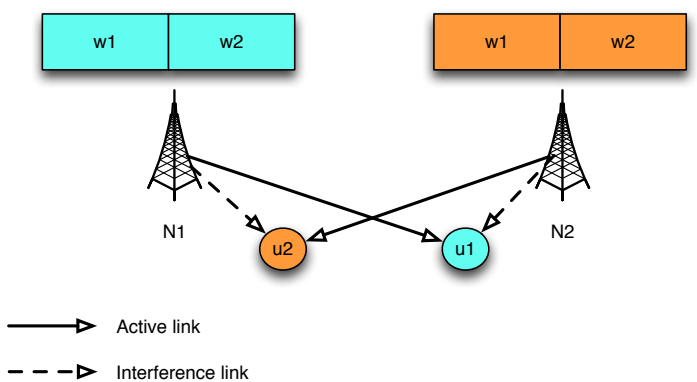

Fig. 4. Two operators (1 and 2) with subscribers (u1,u2) using shared spectrum (w1,w2), yet, not sharing their infrastructure $(\mathrm{N} 1, \mathrm{~N} 2)$.

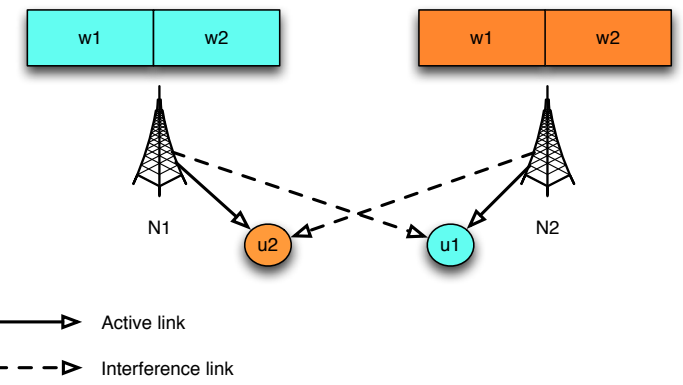

Fig. 5. Two operators (1 and 2) with subscribers (u1,u2) using shared spectrum $(\mathrm{w} 1, \mathrm{w} 2)$ and shared infrastructure $(\mathrm{N} 1, \mathrm{~N} 2)$.

not coordinate or avoid mutual interference. The SINR for this scenario takes the following form:

$$
\mathrm{SINR}=\frac{h_{x} l(x)}{W+\sum_{y \in \Phi \backslash\{x\}} h_{y} l(y)} .
$$

Full sharing scenario In the full sharing scenario operators pool their radio access infrastructure, resulting in network $\Phi=$ $\bigcup_{i \in \mathcal{N}} \Phi_{i}$. In addition, operators also share the spectrum, with joint bandwidth $w=\sum_{i \in \mathcal{N}} w_{i}$. Spectrum pooling results in increased interference as all the base stations are now operating in the same frequency band and users may connect to any of the base stations of the sharing operators, see Fig. 5.

\section{ANALYTICAL RESULTS}

In this section, we apply stochastic geometry to derive analytical expressions for the coverage probability and average user data rate for a shared network created as a union of $|\mathcal{N}|$ networks distributed according to homogeneous Poisson point processes for the three scenarios of infrastructure, spectrum, 
and full network sharing. Firstly, we review existing results for the coverage probability and the average user data rate derived for a non-sharing scenario. Subsequently, we build on those results to derive closed-form expressions for the said metrics for each of our sharing scenarios.

\section{A. Performance metrics}

Coverage probability We define the coverage probability in a cellular network as the probability that a downlink user achieves a target SINR. Therefore, the coverage probability of a shared network can be expressed as:

$$
p_{c}=\mathbb{P}\left(\frac{h_{x} l(x)}{W+\sum_{y \in \Phi^{\prime \prime}} h_{y} l(y)}>\theta\right),
$$

where $x$ is the serving base station chosen from a pool of shared transmitters $\Phi^{\prime}, \Phi^{\prime \prime}$ is the set of transmitters operating in the same spectrum (interferers), and $\theta$ is the reception threshold at the physical layer (assuming a linear receiver with interference treated as noise). Assuming exponential (e.g., Rayleigh) fading and following the derivations in [17], the expression in Eq. (3) can be transformed into:

$$
p_{c}=\int_{0}^{\infty} \exp (-\theta W / \tilde{l}(r)) \mathcal{L}_{I_{r}}(s) p_{r}(r) d r
$$

where $r=\|x\|$ and $\tilde{l}(r) \equiv l(x) ; \mathcal{L}_{I_{r}}(s)=\mathbb{E}_{I_{r}}\left[\exp \left(-s I_{r}\right)\right]$, with $s=\theta / \tilde{l}(r)$, is the Laplace transform of interference $I_{r}$ coming from base stations located at distance at least $r ; p_{r}(r)$ is the distribution of distance to the nearest (serving) base station.

For a non-shared network $\Phi_{i}$ with intensity $\lambda_{i}$ the distribution of distance to the nearest base station and the Laplace transform of interference are known [17], with the latter being expressed as:

$$
\mathcal{L}_{I_{r}}\left(\theta r^{\alpha}\right)=\exp \left(-\pi r^{2} \lambda \rho(\theta, \alpha)\right),
$$

where $\rho(\theta, \alpha)=\theta^{2 / \alpha} \int_{\theta^{-2 / \alpha}}^{\infty} \frac{1}{1+(u)^{\alpha / 2}} d u$. Then, for the case of $\alpha=4, \rho(\theta)=\frac{\pi}{2}-\arctan \left(\theta^{-\frac{1}{2}}\right)$, and assuming no noise, the coverage probability for a non-shared network can be expressed as [17]:

$$
p_{c}^{n s}=\frac{1}{1+\theta^{\frac{1}{2}}\left(\frac{\pi}{2}-\arctan \left(\theta^{-\frac{1}{2}}\right)\right)},
$$

which is a convenient single-parameter representation of coverage in an hPPP-distributed network.

Average data rate We define the average data rate for a typical user in a network of operator $i$ as the rate (expressed in nats/s) of a user when adaptive modulation and coding is set so that Shannon bound ${ }^{6}$ is achieved for the instantaneous SINR of that user. This leads to the following formal definition:

$$
\tau=w_{i} \mathbb{E}\left[\log \left(1+\frac{h_{x} l(x)}{W+\sum_{y \in \Phi^{\prime \prime}} h_{y} l(y)}\right)\right],
$$

\footnotetext{
${ }^{6}$ Real-world mobile systems do not achieve this bound, but to account for this fact would simply require that we rescale our results.
}

where $w_{i}$ denotes the spectrum bandwidth of operator $i$. Then, the average user data rate for a non-shared network $\Phi_{i}$ with intensity $\lambda_{i}$ can be expressed as [17][Theorem 3]:

$$
\begin{array}{r}
\tau=w_{i} \int_{0^{+}}^{\infty} p_{r}(r) \int_{0^{+}}^{\infty} \exp \left(-r^{\alpha} W(\exp (\theta)-1)\right) \\
\cdot \mathcal{L}_{I_{r}}\left(r^{\alpha}(\exp (\theta)-1)\right) d \theta d r
\end{array}
$$

where

$$
\begin{aligned}
\mathcal{L}_{I_{r}}\left((\exp (\theta)-1) r^{\alpha}\right)= & \exp \left(-\pi \lambda_{i} r^{2}(\exp (\theta)-1)^{2 / \alpha}\right. \\
& \left.\cdot \int_{(\exp (\theta)-1)^{-2 / \alpha}}^{\infty} \frac{1}{1+u^{\alpha / 2}} d u\right) .
\end{aligned}
$$

Similarly to the coverage probability, when no noise, with $\alpha=$ 4 , is assumed, this expression gets simplified to [17]:

$$
\int_{0^{+}}^{\tau^{n s}}=w_{i} \frac{1}{1+(\exp (\theta)-1)^{\frac{1}{2}}\left(\frac{\pi}{2}-\arctan \left((\exp (\theta)-1)^{-\frac{1}{2}}\right)\right)} d \theta
$$

\section{B. Derived closed-form expressions}

As sharing may affect the distribution of potential serving base stations as well as the distribution of potential interferers, the terms expressing the distribution of distance to the nearest base station and the Laplace transform of interference may also change. Therefore, in the following we derive expressions for coverage probability and average data rate in the cases of spectrum, infrastructure, and full sharing between $|\mathcal{N}|$ operators with independently distributed radio access infrastructure.

\section{Full sharing scenario}

Proposition 1: The coverage probability of the full sharing scenario can be expressed as in Eq. (6), i.e., $p_{c}^{f s}=p_{c}^{n s}$, while the average user data rate as $\tau^{f s}=\frac{\sum_{i \in \mathcal{N}} w_{i}}{w_{i}} \tau^{n s}$.

Proof: Let us first recall the following result: the superposition of hPPPs is also an hPPP, with the intensity equal to the sum of the intensities of the component processes [18][Proposition 1.3.3]. In the case of the full sharing scenario, the users connect to the closest base station of any of the networks of the sharing operators and suffer interference from all the base stations. Therefore shared network performance corresponds to the performance of a network described as an hPPP $\Phi=\bigcup_{i \in \mathcal{N}} \Phi_{i}$ with intensity $\lambda=\sum_{i \in \mathcal{N}} \lambda_{i}$. Since, for the case of interest, both the coverage probability and the average user data rate are independent from the process intensity, the coverage probability will be as given in Eq. (6), while the data rate will simply be the total bandwidth of the aggregate spectrum multiplied by the integration term in Eq. (9).

\section{Infrastructure sharing scenario}

Theorem 1: The coverage probability for operator $\mathrm{i}$ in the infrastructure sharing scenario can be expressed as:

$$
p_{c}^{i s}(\theta)=\sum_{i \in \mathcal{N}} \frac{\lambda_{i}}{\lambda+\lambda_{i} \theta^{\frac{1}{2}}\left(\frac{\pi}{2}-\arctan \left(\theta^{-\frac{1}{2}}\right)\right)},
$$


while the data rate of a user belonging to operator $i$ as:

$$
\tau^{i s}=w_{i} \int_{0^{+}}^{\infty} p_{c}^{i s}(\gamma) \exp (-\log (\gamma-1)) d \gamma
$$

Proof:

The proof is provided in Appendix A.

\section{Spectrum sharing scenario}

Theorem 2: The coverage probability of a user belonging to operator $i$ in the spectrum sharing scenario can be expressed as:

$p_{c}^{s s}(\theta)=\frac{\lambda_{i}}{\lambda_{i}+\lambda_{i} \theta^{\frac{1}{2}}\left(\frac{\pi}{2}-\arctan \left(\theta^{-\frac{1}{2}}\right)\right)+\frac{\pi}{2} \theta^{\frac{1}{2}} \sum_{j \in \mathcal{N} \backslash\{i\}} \lambda_{j}}$,

while the data rate as:

$$
\tau^{s s}=\left(\sum_{j \in \mathcal{N}} w_{j}\right) \int_{0^{+}}^{\infty} p_{c}^{s s}(\gamma) \exp (-\log (\gamma-1)) d \gamma
$$

\section{Proof:}

The proof is provided in Appendix B.

Before we move on to discuss the numerical results, we cross-validate the correctness of the derived closed-form expressions and our simulations. For that purpose we compare our closed-form expressions to the Monte Carlo simulation results for the hPPP. We performed this comparison for two and more operators, with evenly and non-evenly-sized networks. In Fig. 6 we present just the results for the coverage probability of a two-operator case. As we can see from the figure our closed-form expressions tightly match the results from our simulations.

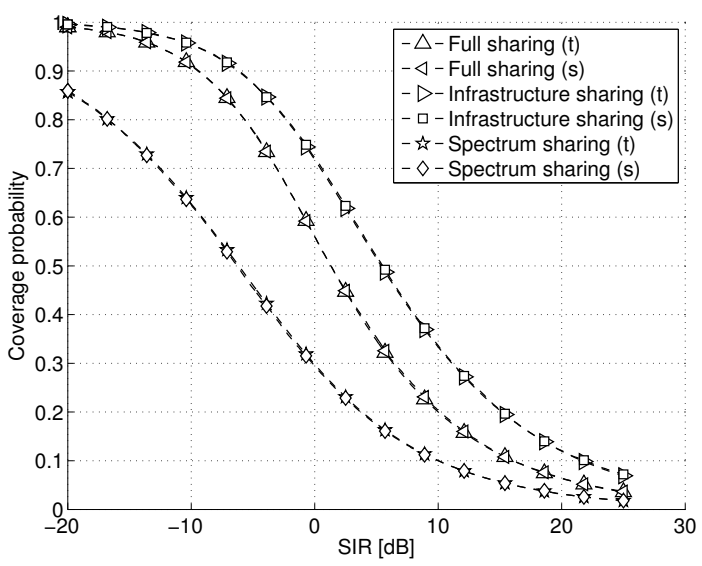

Fig. 6. Cross-validation of the simulations and the derived closed-form expressions for the coverage probability, for a two-operator case where networks of the sharing operators are evenly-sized; " $t$ " - denotes analytical result, and "s" - denotes simulation result.

\section{NumericAl RESUltS AND DISCUSSION}

This section presents numerical results for coverage probability and data rate for different infrastructure deployment models (independent, clustered, co-located) and sharing scenarios (infrastructure, spectrum, and both). In our numerical results we assume that bandwidths of sharing operators, are normalized and equal. In other words, the two operators hold equivalent spectrum licenses. The extension to the case where bandwidths of the two operators are not the same can simply be obtained through linear transformations of our results.

\section{A. Coverage}

The first thing we analyze is the comparative coverage results for various sharing scenarios, when networks of the sharing operators are evenly-sized. In Figures 7(a) to 7(c) we immediately see that infrastructure sharing provides superior coverage as compared to spectrum and full sharing scenarios. This is related to the fact that with the increase in radio access infrastructure sharing, we also shorten the distance to the potential serving base station, i.e., we increase the strength of the signal provided by the serving base station. This effect does not occur when both operators co-locate, as any additional shared base stations, will be co-located with the private ones.

In Fig. 7(b) we observed the coverage performance for a fixed level of clustering, corresponding to the normalized cluster radius of 0.1 (normalized to the size of the simulation window). Now, we change our perspective and observe the impact that clustering of infrastructure has on different sharing scenarios. Figures 8 (a) to 8 (c) present the coverage performance for each of our sharing scenarios when the infrastructure is: independently distributed (ppp), co-located $(u \rightarrow 0)$, and clustered with a variable cluster radius $(u \in\{0.5$, etc. $\}$ ).

As expected, in Figures 8(a) and 8(b) we observe that the increase in clustering (decrease in cluster radius) deteriorates coverage (the direction of change marked with a red arrow) for both full and infrastructure sharing scenarios. In the case of spectrum sharing (Fig. 8(c)) the impact of clustering on the sharing performance is more complex. At low SIR values we observe that the co-located infrastructure provides superior coverage while the independently distributed infrastructure fares the worst. After the cross-over point (slightly below $0 \mathrm{~dB}$ ) the situation reverses. This is related to an interplay between intra- and inter-cluster interference. Clearly, decreasing the cluster radius increases intra-cluster interference. However, as the cluster radius is decreased so does the inter-cluster interference, as the distribution of distances to the interferers outside of the serving cluster changes (pairs of interferers are getting closer to each other). Effectively, while we observe that clustering reduces the probability of obtaining SIR-values above the cross-over point (due to increased inter-cluster interference), we also observe an increase in the probability of obtaining SIR-values below the cross-over point (due to reduced intra-cluster interference), as compared to the independently distributed infrastructure.

\section{B. Data rate}

When average user data rate (Fig. 9) is considered, and the cluster radius is large enough, we can observe that the data rate is always improved over the non-sharing scenario, which, 


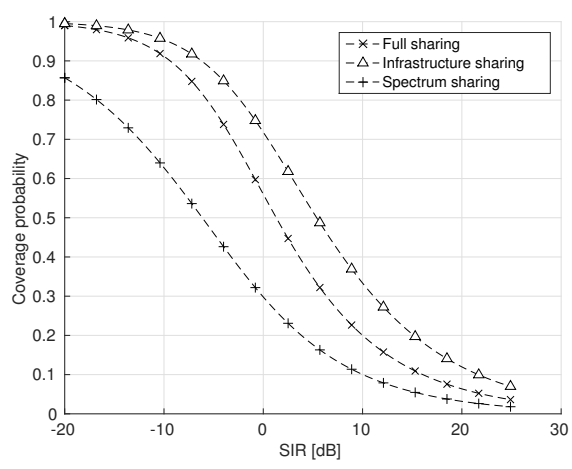

(a)

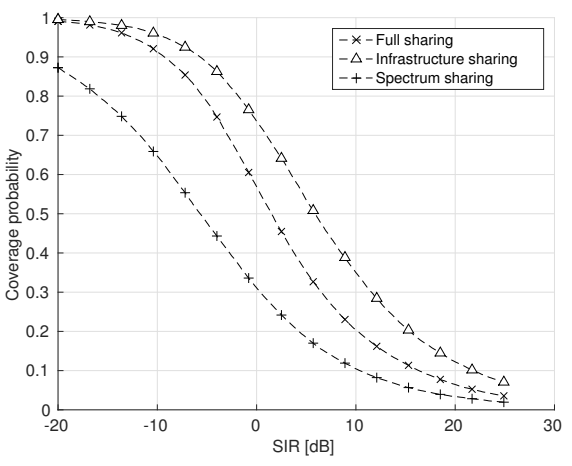

(b)

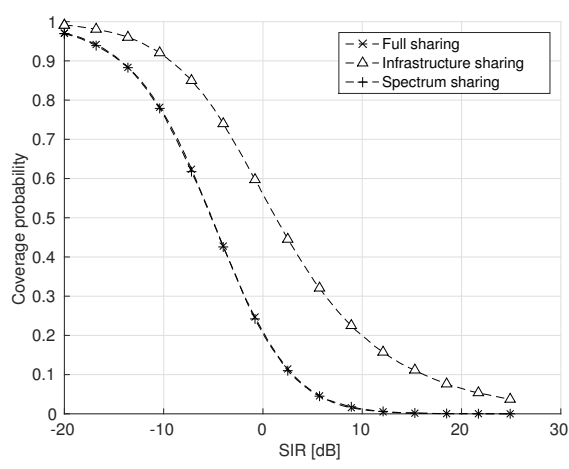

(c)

Fig. 7. The coverage probability for different sharing scenarios for independent (a), clustered (b) and co-located (c) infrastructure.

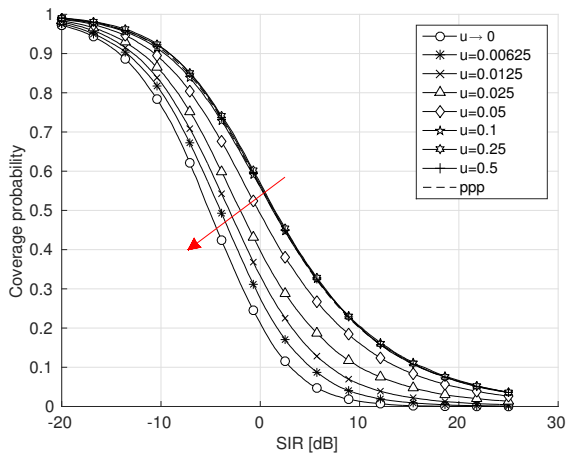

(a)

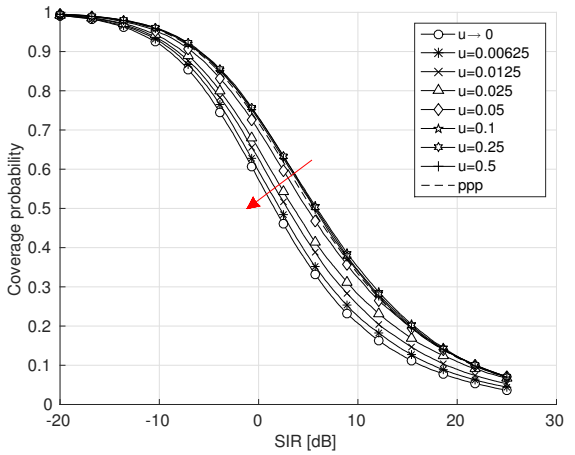

(b)

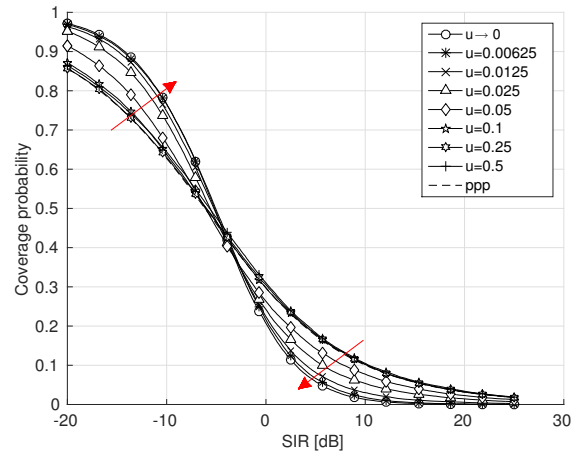

(c)

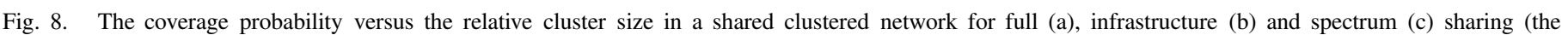
performance of independently distributed network is marked with dashed horizontal line).

as derived in [17], is approximately 1.49 nats/s/Hz. Moreover, full network sharing outperforms all other sharing scenarios for most of the cluster radii. It is only at small cluster radii when the data rate significantly drops, and, in fact, when the infrastructure co-locates full sharing achieves the same performance as the spectrum sharing scenario. From the figure we can also see that significant clustering and co-location of operator networks have a dramatic effect on the data rate performance of any scenario that relies on spectrum sharing (due to strong interference present in the shared network). This result shows the need to apply smart spectrum resource allocation techniques whenever a high level of clustering is present in a multi-operator network, which is typically the case with real mobile networks [14].

\section{Density imbalance between sharing operators}

We now show the performance of network sharing depending on the imbalance in the density of the networks of two operators. We specifically look at the average user data rate. We define the imbalance as the ratio between the respective intensities of their networks $\lambda_{2} / \lambda_{1}$, with 0.1 meaning highly unbalanced networks and 1 standing for evenly-sized networks. We can immediately note from Fig. 10(a) that the imbalance has no impact on the performance of independently distributed networks for the cases when infrastructure is shared, which is precisely the consequence of the results stated in Property 1 and Theorem 1, whereby the density of the infrastructure has no impact on the SIR performance. We can also observe that,

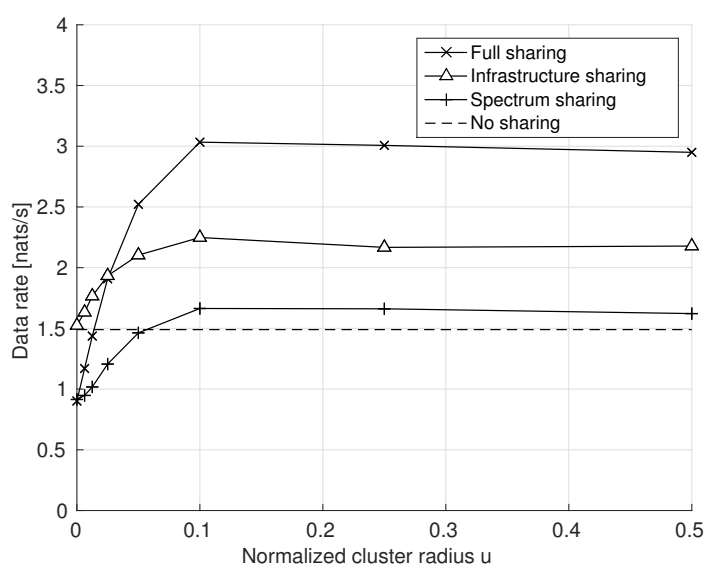

Fig. 9. The average user data rate performance versus the relative cluster size in a shared clustered network, with the performance of a single operator (non-shared) network marked with the a dashed line.

in the case of spectrum sharing, the operator that has a denser network benefits the most from sharing, which is consistent with the fact that there is less interference created by the network of the smaller operator. Interestingly at a specific imbalance level, for the smaller operator it does not make sense to share the spectrum anymore, at least without sharing the infrastructure. In the case of clustered deployment in Fig. 10(b), we observe that the infrastructure sharing gains provide additional performance benefits as soon as the network of 
the smaller operator is enlarged. Interestingly, spectrum sharing for the larger operator outperforms infrastructure sharing when the imbalance is significant, which is related to the minuscule level of interference suffered from the smaller operator. When we look at the imbalance of a co-located network, we can see fast degradation of performance for the spectrum sharing cases, which is again expected as increased co-location produces stronger interfers in the proximity of the desired transmitters.

\section{CONCLUSION}

In this paper, we have assessed the fundamental tradeoffs between spectrum and infrastructure sharing in wireless mobile networks at various degrees of spatial correlation between the networks of sharing operators. What we have observed is that each type of sharing has its own distinctive trait, which we summarize in Fig. 1. While spectrum sharing alone provides an increase in the achievable data rate (at the expense of coverage), infrastructure sharing gives additional degrees of freedom when choosing the point of attachment to the network, resulting in improved coverage. When the two sharing types are combined, we observe significant increase in the average user data rate and improvements to the network coverage as compared to the non-sharing scenario (yet, lower coverage than in the pure infrastructure sharing scenario). Consequently, infrastructure and spectrum sharing cannot be simply substituted for each other, as there exists a tradeoff in the coverage and data rate performance between the two. Moreover, combination of the two approaches does not linearly scale the respective coverage and data rate gains; yet, it provides respectable improvement to both over the non-sharing case, which together with the potential for cost reductions may make it a commercially attractive solution.

Additionally, we observed that the relative spatial distribution, and the relative density of the networks of the sharing operators, have significant impact on the performance, and need to be taken into account when evaluating the potential benefits of network sharing. In particular, when the networks of two mobile operators are closely spatially correlated, then any gains obtained from aggregating spectrum are significantly reduced due to increase in interference. This result, in particular, also stresses the importance of smart (both centralized and distributed) resource management (scheduling, channel assignment, etc.) mechanisms to counter-act the potential increase in interference due to spectrum sharing. Also, the imbalance in the density of the networks of the sharing operators has a significant impact on how the two operators perceive sharing gains. Counter-intuitively, it is the larger operator that gets the most benefits from spectrum sharing, in terms of user data rate. The improvements to network coverage through infrastructure sharing are the highest when the networks of the two operators are similarly-sized.

We believe that this work is a comprehensive introduction to the larger discussion on trade-offs and efficiencies involved in various forms of mobile network sharing and ownership, especially concerning the spatial distribution of base stations involved. An immediate extension of the work involves studying the impact of coordinated spectrum sharing techniques on the performance and trade-offs observed. A broader, multidisciplinary study could also consider the relationship between the economic aspects of various forms of wireless network sharing, such as costs and revenues, and the spatial distribution of a shared network.

\section{ACKNOWLEDGMENT}

This material is based in part upon work supported by the Science Foundation Ireland under grants no. 10/CE/I1853 and 10/IN.1/3007.

\section{APPENDIX A \\ PROOF OF THEOREM 1}

In the infrastructure sharing scenario the point process that describes the serving base station is $\Phi$ with intensity $\lambda=$ $\sum_{i \in \mathcal{N}} \lambda_{i}$. Then, the probability of finding the closest base station at distance $r$ is [19]:

$$
p_{r}(r)=2 \pi \lambda r \exp \left(-\lambda \pi r^{2}\right) .
$$

The interference to which the user is exposed in the infrastructure sharing scenario depends on the network to which the user is connected. Since, in general, we have $|\mathcal{N}|$ operators sharing their networks, we may describe the resulting interference as the expected interference over all possible realizations of interference produced by the networks of individual operators $i \in \mathcal{N}$ :

$$
I_{r}=\mathbb{E}\left[I_{r}\left(\Phi_{i}\right)\right]=\sum_{i \in \mathcal{N}} \mathbb{P}\left(r_{i}>r_{j}, \forall j \neq i\right) I_{r}\left(\Phi_{i}\right), \quad \forall i \in \mathcal{N},
$$

where $I_{r}\left(\Phi_{i}\right)$ denotes the interference of network $\Phi_{i}$ when a user is connected to its closest base station. Since we consider worst case interference conditions, the interference coming from the networks of different operators can be considered independent (no joint resource allocation). Then, the joint probability in Eq. (15) can be found by integrating over the product of all individual probabilities, which given the expression in Eq. (14), will yield:

$$
\mathbb{P}\left(r_{i}>r_{j}, \forall j \neq i\right)=\frac{\lambda_{i}}{\sum_{j \in \mathcal{N}} \lambda_{j}} .
$$

The special case of this result for two operators was derived in [20]. Substituting Eq. (16) into Eq. (15) and taking the Laplace transform of interference yields the following representation:

$$
\mathcal{L}_{I_{r}}(s)=\sum_{i \in \mathcal{N}} \frac{\lambda_{i}}{\sum_{j \in \mathcal{N}} \lambda_{j}} \mathcal{L}_{I_{r}\left(\Phi_{i}\right)}(s) .
$$

Now, the coverage probability can be found by inserting Eq. (14) and Eq. (17), with the Laplace transform for interference in a single-operator network as in Eq. (5) and $v \rightarrow r^{2}$, into the general expression in Eq. (4), which results in:

$$
\begin{aligned}
p_{c}^{i s}=\pi \int_{0^{+}}^{\infty} \exp ( & \left.-\theta v^{\alpha / 2} W\right) \exp (-\lambda \pi v) \\
& \cdot\left(\sum_{i \in \mathcal{N}} \lambda_{i} \exp \left(-\pi \lambda_{i} v \rho(\theta, \alpha)\right)\right) d v .
\end{aligned}
$$

When no noise ( $W=0$ ), with $\alpha=4$, is assumed, similarly to the non-shared case in Eq. (6), our result can be substantially simplified resulting in Eq. (10), which concludes the proof for the coverage probability. 


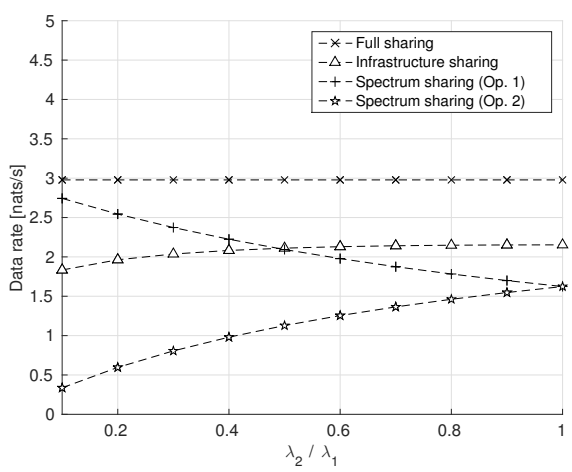

(a)

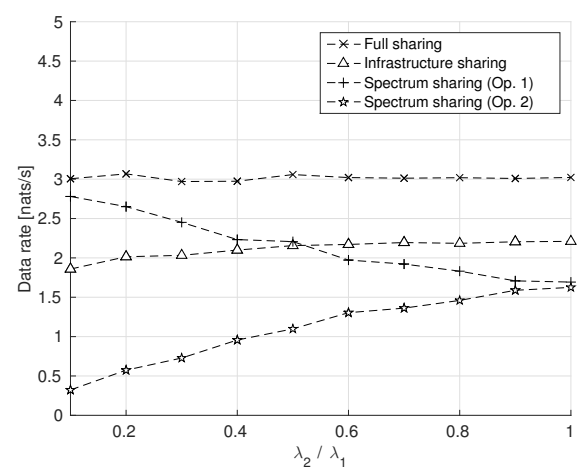

(b)

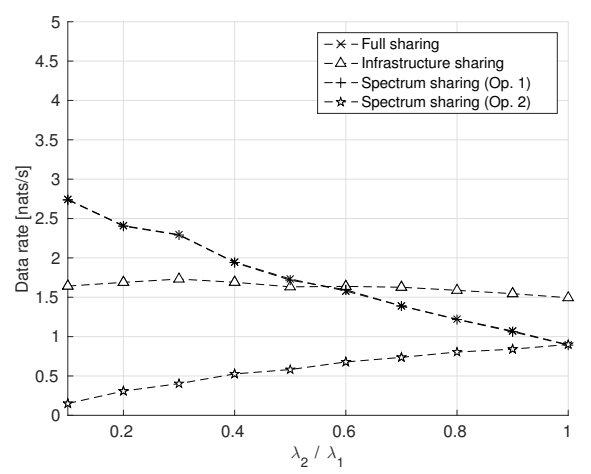

(c)

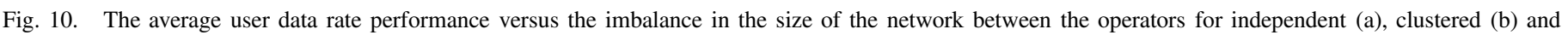
co-located (c) infrastructure.

The derivation of the average user data rate mirrors that of the coverage probability, and relies on plugging Eq. (14) and Eq. (17) into Eq. (8). As in the non-shared case in Eq. (9), taking additional assumptions of $W=0$ and $\alpha=4$ yields the final expression in Eq. (11).

\section{APPENDIX B \\ PROOF OF THEOREM 2}

Let us start by observing that the point process describing the closest base station of operator $i$ subscriber to that operator is $\Phi_{i}$ with intensity $\lambda_{i}$. Therefore, following the same logic as previously, the probability of finding the closest base station at distance $r$ is:

$$
p_{r}(r)=2 \pi \lambda_{i} r \exp \left(-\lambda_{i} \pi r^{2}\right)
$$

The interference to a user of operator $i$ comes in this case from all the base stations of operator $i$ that are at a distance $r$ and further apart, which we denote as $I_{r}\left(\Phi_{i}\right)$. However, due to spectrum sharing there is additional interference which comes from base stations of other operators, whose base stations may be at any arbitrary distance to that user, and we denote this interference as $I_{0}\left(\Phi_{j}\right)$, where $j \neq i$. Now, the total interference in this scenario is $I=I_{r}\left(\Phi_{i}\right)+\sum_{j \neq i} I_{0}\left(\Phi_{j}\right)$, and its Laplace transform can be derived as follows:

$$
\begin{aligned}
\mathcal{L}_{I}(s)= & \mathbb{E}_{I}[\exp (-s I)] \\
= & \mathbb{E}_{I}\left[\exp \left(-s I_{r}\left(\Phi_{i}\right)\right) \exp \left(-s \sum_{j \neq i} I_{0}\left(\Phi_{j}\right)\right)\right] \\
\stackrel{(a)}{=} & \mathbb{E}_{I_{r}\left(\Phi_{i}\right)}\left[\exp \left(-s I_{r}\left(\Phi_{i}\right)\right)\right] \\
& \cdot \prod_{j \neq i} \mathbb{E}_{I_{0}\left(\Phi_{j}\right)}\left[\exp \left(-s \sum_{j \neq i} I_{0}\left(\Phi_{j}\right)\right)\right] \\
= & \mathcal{L}_{I_{r}\left(\Phi_{i}\right)}(s) \prod_{j \neq i} \mathcal{L}_{I_{0}\left(\Phi_{j}\right)}(s),
\end{aligned}
$$

where (a) follows from the worst case scenario interference assumption, which allows us to treat the interference coming from the the networks of different operators as independent, and therefore replace the expectation of the product with the product of individual expectations. Finally, when we plug Eq. (5) and $\mathcal{L}_{I_{0}\left(\Phi_{j}\right)}\left(\theta r^{\alpha}\right)=\exp \left(-\pi r^{2} \lambda \rho_{0}(\theta, \alpha)\right)$, where $\rho_{0}(\theta, \alpha)=\theta^{2 / \alpha} \int_{0^{+}}^{\infty} \frac{1}{1+(u)^{\alpha / 2}} d u$, into Eq. (20), we get the following expression:

$$
\mathcal{L}_{I}\left(\theta r^{\alpha}\right)=\exp \left(-\pi r^{2}\left(\lambda_{i} \rho(\theta, \alpha)+\rho_{0}(\theta, \alpha) \sum_{j \neq i} \lambda_{j}\right)\right) .
$$

Now, plugging the obtained Laplace transform together with Eq. (19) into Eq. (4), and making substitution $v \rightarrow r^{2}$, yields the following expression for the coverage probability of operator $i$ :

$$
\begin{aligned}
p_{c}^{s s}= & \pi \lambda_{i} \int_{0^{+}}^{\infty} \exp \left(-\theta v^{\alpha / 2} W\right) \exp \left(-\lambda_{i} \pi v\right) \\
& \cdot \exp \left(-\pi v\left(\lambda_{i} \rho(\theta, \alpha)+\rho_{0}(\theta, \alpha) \sum_{j \neq i} \lambda_{j}\right)\right) d v .
\end{aligned}
$$

Similarly to the infrastructure sharing scenario, when no noise, with $\alpha=4$, is assumed the above expression simplifies to Eq. (12).

The derivation of the average user data rate mirrors that of the coverage probability.

\section{REFERENCES}

[1] P. Di Francesco, F. Malandrino, and L. A. DaSilva, "Mobile Network Sharing Between Operators: A Demand Trace-driven Study," in Proceedings of the 2014 ACM SIGCOMM Workshop on Capacity Sharing Workshop, August 2014, pp. 39-44.

[2] M. Nawrocki, H. Aghvami, and M. Dohler, Understanding UMTS Radio Network Modelling, Planning and Automated Optimisation: Theory and Practice. John Wiley \& Sons, 2006.

[3] T. Frisanco et al., "Infrastructure Sharing and Shared Operations for Mobile Network Operators: From a Deployment and Operations View," in IEEE International Conference on Communications (ICC), May 2008, pp. 2193-2200.

[4] J. Kibiłda and L. A. DaSilva, "Efficient Coverage Through Interoperator Infrastructure Sharing in Mobile Networks," in Proc. of IFIP Wireless Days, November 2013, pp. 1-6.

[5] (2012, February) Denmark: Network Sharing Agreement in Danish Mobile Telecommunications Sector. [Online]. Available: http://ec. europa.eu/competition/ecn/brief/02_2012/dk_mobile.pdf

[6] C. Gabriel. (2011, July) Orange and TMo merge networks in Poland. Rethink Wireless. [Online]. Available: http://www.rethink-wireless. com/2011/07/22/orange-tmo-merge-networks-poland.htm

[7] L. Doyle et al., "Spectrum without Bounds, Networks without Borders," Proceedings of the IEEE, vol. 102, no. 3, pp. 351-365, March 2014. 
[8] U. Paul et al., "Understanding traffic dynamics in cellular data networks," in Proceedings IEEE INFOCOM, April 2011, pp. 882-890.

[9] M. Matinmikko et al., "Spectrum sharing using licensed shared access: the concept and its workflow for LTE-advanced networks," IEEE Wireless Communications, vol. 21, no. 2, pp. 72-79, April 2014.

[10] "TS 23.251 V10.4.0; Universal Mobile Telecommunications System (UMTS); LTE; Network sharing; Architecture and functional description," 3GPP, Tech. Rep., 2010.

[11] E. A. Jorswieck et al., "Spectrum Sharing Improves the Network Efficiency for Cellular Operators," IEEE Communications Magazine, vol. 52, no. 3, pp. 129-136, March 2014.

[12] J. S. Panchal, R. D. Yates, and M. M. Buddhikot, "Mobile Network Resource Sharing Options : Performance Comparisons," IEEE Transactions on Wireless Communications, vol. 12, no. 9, pp. 4470-4482, September 2013.

[13] M. Michalopoulou, J. Riihijarvi, and P. Mahonen, "Studying the Relationships between Spatial Structures of Wireless Networks and Population Densities," in 2010 IEEE Global Telecommunications Conference (GLOBECOM), December 2010, pp. 1-6.

[14] J. Kibiłda, B. Galkin, and L. A. DaSilva, "Modelling Multi-operator Base Station Deployment Patterns in Cellular Networks," (under review).

[15] M. Haenggi, Stochastic Geometry for Wireless Networks. Cambridge University Press, 2013.

[16] A. Guo and M. Haenggi, "Spatial Stochastic Models and Metrics for the Structure of Base Stations in Cellular Networks," IEEE Transactions on Wireless Communications, vol. 12, no. 11, pp. 5800-5812, November 2013.

[17] J. G. Andrews, F. Baccelli, and R. K. Ganti, "A tractable approach to coverage and rate in cellular networks," IEEE Transactions on Communications, vol. 59, no. 11, pp. 3122-3134, November 2011.

[18] F. Baccelli and B. Błaszczyszyn, Stochastic geometry and wireless networks: Volume 1: Theory. Now Publishers Inc, 2009, vol. 1.

[19] M. Haenggi, "On distances in uniformly random networks," IEEE Transactions on Information Theory, vol. 51, no. 10, pp. 3584-3586, October 2005.

[20] S. Hua, P. Liu, and S. S. Panwar, "The urge to merge: When cellular service providers pool capacity," in IEEE International Conference on Communications (ICC), June 2012, pp. 5020-5025. 